\title{
Investigación acción y desarrollo docente en las escuelas normales mexicanas
}

\section{Action research and professional development in Mexican teachers' colleges}

\section{DOI: https://doi.org/10.32870/dse.v0i21.606}

Jesús Izquierdo Sandoval*

Silvia Patricia Aquino Zúñiga**

Verónica García Martínez***

María Luisa Ramón Rodríguez ${ }^{* * * *}$

\begin{abstract}
Resumen
Diversos estudios han explorado los beneficios y desafíos en la implementación de la investigación-acción para el desarrollo docente en diversos niveles educativos. No obstante, se requieren indagaciones con los formadores de los futuros docentes de educación básica. Empleando un diseño interpretativo de casos, este estudio explora el conocimiento, experiencias, expectativas y retos de los profesores en escuelas normales públicas cuando la investigación-acción se presenta como una estrategia institucional para el desarrollo docente. En el estudio, participaron profesores de tres escuelas normales del sureste mexicano. El análisis de agregación categórica con datos de encuestas, talleres, grupos focales y entrevistas revela homogeneidad en la percepción de los beneficios de la investigación-acción en los profesores. Sin embargo, los profesores carecen de experiencia y claridad conceptual y metodológica. Aunado a esto, deben vencer diversos desafíos personales e institucionales para emplear la investigación-acción como una estrategia institucional de desarrollo docente.
\end{abstract}

Palabras clave: Educación normalista - formación de formadores - investigación acción.

\begin{abstract}
Numerous studies have explored the benefits and challenges in the implementation of action research for teacher development in various educational levels. Nevertheless, research is needed on the educators who train the future teachers of public elementary education. Through an interpretative collective case
\end{abstract}

* Doctor en Educación en Segundas. SNI 1. Cuerpo Académico Consolidado: Investigación e Innovación Educativas. Profesor Titular de la Universidad Juárez Autónoma de Tabasco. México. jesus.izquierdo@mail.mcgill.ca

** Doctora en Ciencias de la Educación. Línea de investigación: Investigación e Innovación Educativa. Proyectos de investigación desarrollados sobre inclusión educativa de ciegos en educación superior; evaluación docente, de instituciones y del aprendizaje. Profesora-investigadora de la División Académica de Educación y Artes de la Universidad Juárez Autónoma de Tabasco. México. saquinozuniga@gmail.com

*** Doctora en Ciencias Sociales. SNI 1. Líneas de investigación: tecnología, medios de comunicación y educación. Profesora Titular de la Universidad Juárez Autónoma de Tabasco. México.veronica.garcia@ujat.mx

**** Licenciada en Ciencias de la Educación. Colaboradora del Cuerpo Académico en Formación: Evaluación Educativa. Profesora de tiempo completo en la Escuela Normal de Educación Primaria “Rosario María Gutiérrez Eskildsen". Tabasco. México. ramon.mluisa@hotmail.com 
study, this research explored the knowledge, experience, expectations and challenges of teacher educators when they are required to implement action research as an institutionalized professional development strategy. Teacher educators from three teachers' colleges in southeastern Mexico participated in the study. The results of categorical aggregation analyses conducted on surveys, workshop, focus group, and interview data reveal homogeneous perceptions about the use of action research among the participants, although they lack previous experience with this research type as well as conceptual and methodological clarity. Moreover, they must overcome different personal and institutional challenges to implement action research as an institutional professional teacher development strategy.

Keywords: Teachers' colleges - teacher educators - action research.

\section{Introducción}

Ante las constantes reformas educativas, el desarrollo del profesorado constituye un reto imperante en los sistemas educativos. Si bien, para tal efecto existen diversas opciones, en la actualidad se valoran principalmente los procesos formativos que empoderan al docente y lo convierten en un agente de cambio capaz de identificar retos de su contexto, documentarse, implementar y evaluar acciones para superarlos (Arias y Restrepo, 2009; Imbernón, 2000; Kayaoglu, 2015). Desde esta mirada, la investigación-acción (IA) constituye una alternativa para que el docente entre en un proceso de mejora permanente (Latorre, 2003; Elliot, 2005; Flessner y Stuckey, 2014).

Durante la IA, el profesor se ve inmerso en un proceso de reflexión permanente que le ayuda a articular aspectos teóricos, axiológicos y ontológicos de su labor educativa para subsanar problemáticas empleando prácticas trasformadoras (Latorre, 2003; Elliot, 2005; Becerra y Moya, 2010). Para ello, el profesor colabora con sus pares, se documenta, organiza prácticas innovadoras y analiza evidencias (Arias y Restrepo, 2009). En estas acciones, el docente desarrolla principios para sistematizar el proceso reflexivo (Luttenberg, Meijer y Oolbekkink-Marchand, 2016).

Por estos y otros beneficios, instituciones educativas internacionales han establecido políticas que fomentan el empleo de la IA como una estrategia para el desarrollo docente y la mejora educativa (Flessner y Stuckey, 2014; Foreman-Peck y Murray, 2008; loannidou y Patsalidou, 2015; Kayaoglu, 2015). En México, en la Educación Normal, las reformas al Plan de Estudios de la Licenciatura en Educación Primaria y Preescolar han manifestado interés en promover la IA en los futuros docentes de Educación Básica. En estas reformas, la investigación se ha visualizado como una estrategia que permitirá a los futuros docentes desarrollar conocimientos y habilidades que redundarán en la mejora de los procesos educativos de sus contextos.

En estas reformas, sin embargo, no ha habido congruencia entre el objetivo de fomentar la investigación en la formación del futuro docente y los contenidos curriculares que para tal efecto se han planteado. Por ejemplo, el Programa para la Transformación y Fortalecimiento Académico de las Escuelas Normales sostenía que el futuro docente debía desarrollar "disposición y capacidades propicias para la investigación científica, curiosidad y capacidad de observación"

Diólo@os 
(SEP, 1997: 32). Pero, las asignaturas del Plan de Estudios 1997 estaban previstas para el desarrollo de la competencia didáctica, dejando de lado las intenciones en materia de investigación.

Además, la Educación Normal en México no ha consolidado el empleo de la investigación debido a la escasa capacitación de los profesores en el tema en las escuelas normales y a una falta de visión de los profesores para articular la docencia y la investigación (Vilchis, 2015). En este tenor, en 2017 la Dirección General de Educación Superior (DGESU) y la Dirección General para Profesionales de la Educación Superior (DGESPE) identificaron que los docentes de las escuelas normales requieren de iniciativas que impulsen la articulación entre la docencia y la investigación, el desarrollo docente, y el desarrollo de conocimientos y habilidades en investigación, que redunden en la formación de sus estudiantes (Malo, 2018).

Para impulsar tales cambios, en 2017, la DGESU y la DGESPE pusieron en marcha una estrategia nacional denominada: Red de Comunidades para la Renovación de la EnseñanzaAprendizaje en la Educación Superior, RECREA (Malo, 2017). Esta red nacional es financiada por el Programa de Desarrollo y Superación Docente (PRODEP). El propósito de la red es promover una cultura de colaboración para la innovación y mejora de las prácticas docentes entre los profesores en Cuerpos Académicos en Formación (CAF), en las Escuelas Normales, y profesores en Cuerpos Académicos en Consolidación (CAeC) o Consolidados (CAC) en universidades estatales.

En la RECREA, la innovación y mejora de las prácticas docentes se centra en la recuperación de las prácticas innovadoras de los docentes normalistas considerando tres ejes fundamentales: el pensamiento complejo, la investigación-acción y el uso de las tecnologías y la información (TIC) en el aula, donde la planeación del curso resulta fundamental. En el caso de la IA, esta debe promover el proceso reflexivo para que el docente normalista recupere sus prácticas innovadoras, y resuelva situaciones o problemáticas del aula. Además, la IA debe permitirles fortalecer sus competencias investigativas y sus perfiles profesionales, para generar conocimientos y productos científicos que incidan en el nivel de consolidación de sus cuerpos académicos (CA).

En el marco de la iniciativa RECREA, este estudio se desarrolló con el objetivo de documentar los conocimientos, las experiencias, las expectativas y los retos de los profesores en las escuelas normales del sureste mexicano con respecto al empleo de la IA como una estrategia institucional de desarrollo docente.

\section{Investigación-acción y desarrollo docente}

Diversos investigadores señalan que la IA en los sistemas educativos puede vislumbrarse con diversas finalidades; estas pueden ir desde un proceso personal de introspección hasta la implementación de una iniciativa institucional (Adler, 2003; Colmenares y Piñero, 2008; Dick, 2010; Vaughan y Burnaford, 2015). Estas finalidades repercuten no solo en el enfoque y alcance de la IA. También redundan en la perspectiva epistemológica y los fundamentos metodológicos de su aplicación. Basados en la discusión presentada por Foreman-Peck y Murray (2008: 148-150) 
y Elliot (2005), este escrito asume la IA como una estrategia para el desarrollo docente bajo la concepción de practical phylosophy.

Desde esta concepción, la IA permite al docente entrar en un proceso de desarrollo que, de manera colaborativa, subsana problemáticas a través de prácticas educativas que trasforman su contexto educativo. En tal exploración, mediante la autoevaluación y la reflexión, el docente reconstruye sus principios teóricos, conocimientos investigativos y valores educativos para la documentación y el análisis sistemático de lo que ocurre en su entorno. Para tal efecto, entra en un ciclo donde identifica la problemática, establece un plan de acción, lo implementa, monitorea su impacto, y reinicia el ciclo (véase Latorre, 2003; Adler, 2003; Burns, 2010; Krell y Fichtman, 2012).

El impacto de la IA en el desarrollo docente ha sido investigado en diferentes contextos educativos (Dick, 2010; Vaughan y Burnaford, 2015). En estos contextos, los docentes han empleado la IA para solventar problemáticas en relación con el aprendizaje, sus estrategias didácticas, inequidad social y políticas educativas. Un resultado frecuente es que, al solucionar tales problemáticas, los docentes desarrollan conocimientos, habilidades y actitudes que fortalecen, entre otros aspectos, su práctica educativa, su perfil profesional, la articulación docencia-investigación y su empoderamiento. No obstante, con estos beneficios se presentan retos formativos, e incluso afectivos, que mitigan el potencial de la IA (Krell y Fichtman, 2012; Luttenberg, Meijer y Oolbekkink-Marchand, 2016).

Como lo señala Burns (2010), anterior al empleo de la IA, los docentes conciben su labor educativa estrechamente vinculada con la parte operativa de los procesos de enseñanzaaprendizaje. Si bien, durante tales procesos los docentes cuestionan su práctica educativa, sus cuestionamientos se presentan alejados de la sistematización y la fundamentación (Adler, 2003; Arias y Restrepo, 2009; loannidou y Patsalidou, 2015; Luttenberg, Meijer y Oolbekkink-Marchand, 2016). Ante la falta de sistematización y de competencias para sustentar sus hallazgos (Adler, 2003), los docentes no perciben su labor docente como una oportunidad para generar conocimientos que repercutan más allá de sus aulas o su desarrollo docente (Foreman-Peck y Murray, 2008; Kayaoglu, 2015).

Además de explorar la implementación de la IA para el desarrollo docente en diferentes niveles educativos, los estudios han analizado sus retos y beneficios con diferentes tipos de profesores. En este rubro, las investigaciones se han realizado con profesores en servicio y, en un número menor, con profesores en estudios de posgrado (Vaughan y Burnaford, 2015) o en formación inicial (Ulvik y Riese, 2015). Con estas poblaciones se han obtenido resultados que son consistentes con aquellos presentados en el párrafo anterior (véase Ulvik y Riese, 2015; Vaughan y Burnaford, 2015). No obstante, Tanner y Davis (2008), y Krell y Fichtman (2012) señalan que existen pocos estudios relacionados con el empleo de la IA para fortalecer el desarrollo de los formadores de docentes.

Diólopos sobre Educación año 11 | número 21 | julio-diciembre 2020 | ISSN 2007-2171 
Los formadores de docentes constituyen un grupo esencial de actores educativos, porque, al implementar la IA con su propia práctica educativa, experimentan las tensiones y contradicciones que sus estudiantes-docentes vivirán en la búsqueda de la mejora educativa (Adler, 2003; Krell y Fichtman, 2012). También, desarrollan conocimientos, habilidades y empatía para propiciar y evaluar en los estudiantes-docentes la creatividad, el cuestionamiento, la reflexión y la crítica constructiva durante las diversas etapas de la IA (Adler, 2003; Krell y Fichtman, 2012). Así, cuando los formadores de docentes recurren a la IA para analizar su práctica, ellos y sus estudiantes entran de manera simultánea en un proceso de desarrollo docente (Ulvik y Riese, 2015: 442).

Si bien las secciones anteriores ponen de manifiesto el potencial de la IA para el desarrollo docente, su implementación no es siempre acogida positivamente en los sistemas educativos. Al respecto, Foreman-Peck y Murray (2008) señalan que la IA puede ser percibida como una aliada para reconstruir las prácticas educativas, los perfiles docentes y las políticas institucionales, si su implementación surge como una iniciativa propia de los contextos donde se desarrolla. No obstante, cuando se recurre a ella como una estrategia institucional, sobre todo en sistemas educativos centralizados, puede generar escepticismo, tensiones y contradicciones (ForemanPeck y Murray, 2008; Kayaoglu, 2015).

Las reacciones de rechazo se presentan debido a la experiencia previa de los docentes en trabajos colegiados en sus centros educativos, su antigüedad, su personalidad, su posicionamiento político y el tiempo institucional asignado para la IA (Adler, 2003, Flessner y Stuckey, 2014; loannidou y Patsalidou, 2015). También, el nivel de compromiso de los profesores ante la política institucional genera tensión con administrativos, grupos sindicales y pares docentes (Flessner y Stuckey, 2014; Krell, 2012). Ante estos retos, diversos investigadores sugieren explorar, no solo los conocimientos y experiencias de los docentes en IA sino también las expectativas y desafíos que conlleva su implementación institucionalizada (Adler, 2003; Kayaoglu, 2015; Krell y Fichtman, 2012).

Partiendo de estos referentes, este estudio plantea la siguiente pregunta de investigación: ¿Qué beneficios y desafíos perciben los profesores en las escuelas normales mexicanas para implementar la IA como una estrategia institucional de desarrollo docente en sus contextos educativos? Basados en esta pregunta, el objetivo del estudio fue identificar los conocimientos, las experiencias, las expectativas y los retos de los profesores para implementar la IA como una estrategia institucional.

\section{Método}

Para responder la pregunta de investigación, se empleó un estudio de casos colectivos. El estudio fue de tipo interpretativo para propiciar la interacción directa entre el investigador y el investigado en el contexto de los participantes. Este tipo de interacción permite identificar su- 
cesos y datos relevantes que facilitan la comprensión de lo que se estudia (Balcázar, González, Gurrola y Moysén, 2006; Creswell, 2013; Thouin, 2014).

\section{Contexto y participantes}

Como casos colectivos, se identificó a cada uno de los tres grupos de profesores normalistas de tiempo completo que participaron en RECREA en las escuelas normales del sureste mexicano. Estos profesores se encontraban adscritos a los CAF en una escuela normal de Yucatán y dos escuelas normales de Tabasco. En cada escuela se recolectaron datos de cinco participantes. Para salvaguardar su confidencialidad, en las siguientes secciones se emplean seudónimos, omitiendo la procedencia de los participantes.

Los profesores participaron de enero a julio 2019 en RECREA, donde su desarrollo docente se favoreció mediante la organización de proyectos de IA que les permitieran innovar su práctica educativa, fortalecer su planeación didáctica e integrar las TIC en su labor docente. En la red, los profesores normalistas colaboraron con los otros integrantes de su CA y los autores del estudio.

Por el carácter interpretativo del estudio, la interacción entre investigadores e investigados (Balcázar et al., 2006; Creswell, 2013) ocurrió en las escuelas normales de los participantes. La naturaleza institucionalizada en la implementación de la IA se dio en el marco de RECREA con auspicio y apoyo de la DGESU, la DGESPE y las escuelas normales de los docentes. Esta interacción se dio en etapas que incluyeron, además de comunicación vía telefónica, talleres presenciales para la implementación de un proyecto de IA, la planeación didáctica y el empleo de las TIC, reuniones con colegas del $C A$, seguimiento de las actividades por parte de los investigadores; $y$ el análisis colegiado de sus experiencias en IA.

\section{Instrumentos}

Al inicio de la investigación se definieron tres instrumentos para documentar los conocimientos, experiencias, expectativas y desafíos en la implementación de la IA: una entrevista individualizada, un grupo focal y un taller sobre IA. No obstante, de acuerdo con Latorre (2003), Balcázar et al. (2006), Creswell (2013) y Thouin (2014), conforme evoluciona la interacción investigadorinvestigados, en un estudio interpretativo es adecuado implementar otros instrumentos para profundizar acerca de la realidad de los participantes. Por lo tanto, eventualmente, se incluyó también una segunda entrevista individualizada, un segundo taller, un segundo grupo focal y una encuesta. Los instrumentos fueron aplicados por los investigadores en cada escuela normal.

Encuestas. Este instrumento se diseñó empleando preguntas abiertas con respuestas cortas (Dörney, 2002). Su aplicación permitió recolectar datos sociodemográficos, de formación pro-

Diálo@os sobre Educación año 11 | número 21 | julio-diciembre 2020 | ISSN 2007-2171 
fesional, experiencia docente en la escuela normal de adscripción, experiencia en investigación aplicada, experiencia en investigación-acción y distribución de horas en diferentes actividades (docencia, investigación, comisiones, etc.). Este instrumento no se aplicó para contabilizar la frecuencia de las respuestas sino para obtener información personalizada que permitiera una comprensión de la situación laboral, profesional y docente de cada participante, y generar preguntas personalizadas durante las entrevistas y grupos focales (Creswell, 2013).

Grupos focales. Estos se organizaron considerando el principio metodológico de una discusión informal pero estructurada (Balcázar et al., 2006), con los profesores participantes en cada escuela normal. El objetivo fue explorar los conocimientos, experiencias, expectativas y desafíos en actividades de investigación de manera colegiada. En el primer grupo focal se hicieron cuatro preguntas detonantes relacionadas con la experiencia en proyectos de investigación; la diferencia entre investigación básica, aplicada e IA; experiencia supervisando trabajos de investigación de estudiantes; y experiencia impartiendo asignaturas de investigación.

El segundo grupo focal se realizó para discutir los resultados obtenidos en la primera fase del estudio y asegurar una interpretación apropiada de la realidad de los investigados (Creswell, 2013). En consecuencia, en este grupo se discutieron con los profesores los datos recabados e interpretaciones realizadas posteriores a la primera semana de trabajo, en relación con conocimientos, experiencias, expectativas y desafíos en cada escuela normal para implementar la IA como una estrategia institucional. Además, se discutieron los desafíos presentados en cada escuela normal para organizar un proyecto de IA. Al término de cada grupo focal, se redactó una síntesis de la información vertida por los participantes.

Entrevistas a profundidad. Las entrevistas se desarrollaron de manera individual con los 15 profesores, considerando las pautas metodológicas establecidas por Thouin (2014) y Creswell (2013). Para su aplicación, se desarrolló un guion con preguntas detonantes. Posteriormente, considerando que estas entrevistas permiten un cuestionamiento confidencial e individualizado, se favoreció una interacción bidireccional entrevistado-entrevistador (Balcázar et al, 2006) con cuestionamientos espontáneos relacionados con la realidad de cada participante.

Durante la primera entrevista, realizada en enero, las preguntas detonantes se centraron en la experiencia individual en investigación-acción, los beneficios de la IA en el desarrollo docente y las expectativas para su implementación. Durante la segunda entrevista, realizada en mayo, las preguntas detonantes se centraron en las respuestas de las encuestas y los desafíos personales, institucionales y organizacionales de cada profesor para implementar un proyecto de IA en su escuela normal. Las entrevistas duraron entre 15 y 30 minutos y fueron audiograbadas. De las entrevistas se extrajo información para complementar los datos obtenidos en los otros instrumentos con respecto a las bondades y desafíos en la implementación de la IA. 
Talleres. Los talleres se realizaron partiendo del principio metodológico de que constituyen una técnica de recolección de datos para documentar la reconstrucción de los conocimientos y experiencias de los participantes con respecto al tema de interés (véase Latorre, 2003; Burns, 2010).

El primer taller tuvo una duración de seis horas y fue impartido en el marco de un seminario de cinco días que incluía un total de 30 horas de trabajo presencial. Durante el primer taller, se buscó homologar los conocimientos acerca de la IA de los integrantes en cada escuela normal. En este taller se presentó información acerca de la situación de la IA en las escuelas normales, el papel que desempeña en el marco de RECREA, sus principios y etapas, la recolección de datos y ejemplos de experiencias realizadas por profesores normalistas en Sudamérica y la Península ibérica.

El segundo taller tuvo una duración de doce horas de trabajo presencial. En este taller, en equipos de trabajo, se revisó el proyecto de IA planeado para afrontar una problemática en la escuela normal y se discutieron los desafíos y beneficios de su aplicación. La información se presentó empleando archivos PowerPoint que incluían diapositivas y videos; también se distribuyeron artículos de revistas científicas. Empleando estos materiales se favorecieron discusiones grupales, presentaciones y trabajo en pareja. De los talleres se extrajeron notas con respecto a las bondades y desafíos en la implementación de la IA en las escuelas normales.

\section{Procedimientos}

Primero, los investigadores establecieron un programa de trabajo con los directores de las escuelas normales con apoyo de la DGESU y la DGESPE. Enseguida, se realizaron las visitas a las escuelas normales, con duración de una semana en cada una. En la primera, entre enero y febrero, se llevó a cabo un grupo focal, un taller y entrevistas individualizadas. Finalmente, la segunda visita se realizó en mayo, y durante este tiempo se implementó una encuesta, un grupo focal, un taller y entrevistas individualizadas.

\section{Resultados}

Para responder la pregunta de investigación, considerando el objetivo del estudio, los datos se analizaron empleando la técnica de agregación categórica (Creswell, 2013). Para tal efecto, primero se identificaron las cuatro dimensiones de interés: conocimientos, experiencias, beneficios y desafíos de la IA. Partiendo del principio de agregación (Creswell, 2013), en cada dimensión se agruparon los datos procedentes de los diferentes instrumentos y participantes independientemente del caso al que pertenecían (Creswell, 2013).

Este análisis descarta la identificación de frecuencias de las diversas temáticas al interior de cada dimensión, ya que se perciben como complementarias unas de otras; así, se obtiene una visión holística de los factores que contribuyen en cada dimensión de estudio (Balcázar et al.,

Diólo pos

Sobre Educación año 11 | número 21 | julio-diciembre 2020 | ISSN 2007-2171 
2006; Burns, 2010; Thouin, 2014). A continuación, se presentan las interpretaciones para cada dimensión.

\section{Conocimientos de investigación}

A través del proyecto se documentó la necesidad expresa de los profesores de fortalecer sus conocimientos y habilidades para la investigación. Durante los talleres, entrevistas y grupos focales, los docentes de las tres escuelas externaron de manera sistemática dos principios centrales de la IA: la identificación de un problema de investigación y la necesidad de solucionar la problemática. Como lo ilustran los comentarios de Salvador, un aspecto evidente de la IA para los docentes es su contribución a la mejora educativa.

Salvador: para mí la investigación acción es un proceso de indagación, un proceso de búsqueda que emprende o que puede emprender a un grupo de personas con la idea de encontrar o de buscar problemáticas sociales, que al intervenir de manera grupal pueden generar algunas soluciones colectivas que mejoren a la sociedad.

Algo evidente fue que existe opacidad en relación con los fundamentos que distinguen a la IA de otros tipos de investigación educativa. Gimena, por ejemplo, establece que en sus proyectos de IA no logra cubrir los criterios "del método científico," pero sí reflexiona acerca del impacto del proyecto en los aprendizajes de sus estudiantes.

Gimena: [...] Eh, pues así objetivamente que yo desarrolle un proyecto [de investigación acción] a base, con los pasos del método científico, no; pero implícitamente sí, porque cada vez que realizo un trabajo reflexiono acerca de lo que estoy haciendo bien, de las dificultades que tengo, de los aprendizajes logrados.

Un aspecto notorio en los datos fue la heterogeneidad en el conocimiento acerca de la investigación entre los docentes de las tres escuelas, y al interior de cada escuela normal. Martha, por ejemplo, al explicar un proyecto que realiza su CA, ilustra aspectos de la IA como la identificación de una problemática, el marco epistemológico de tal problemática y las actividades del plan de acción. Los conocimientos de Martha contrastan con los de Salvador, quien también participa en el proyecto.

Martha: Estamos trabajando con un proyecto [...] acerca de la importancia de la socioafectividad en los procesos de enseñanza aprendizaje, tenemos problemas de bullying, porque la socioafectividad está... muy descuidada; $[\ldots]$ y entonces a partir de esto, surge este proyecto para dar una [...] solución; [...] 
hemos sensibilizado a los padres de familia [...] la importancia que tiene sumarse al esfuerzo del trabajo para beneficio de la formación del niño, no divorciarse [...] ahí es donde va esa propuesta.

Salvador: el proyecto, ahora con lo del cuerpo académico [...] estoy llevando uno, [...] ahora viendo el concepto de investigación acción, posiblemente esté enmarcado dentro de esa línea, pero que te pueda definir que esté encaminado hacia investigación acción no te lo puedo confirmar porque, tengo que ser honesto... estamos haciendo investigación y estamos siendo apoyados por una investigadora, pero hasta ahí.

Al confrontar a los participantes con estos resultados durante el segundo grupo focal, como lo ilustran los siguientes comentarios de Salvador y varios docentes, los profesores normalistas reconocen que, independientemente de su grado de estudios, carecen de conocimientos y habilidades en investigación.

Salvador: No tenemos la formación en investigación y eso creo que es una limitante fuerte que tenemos aquí, yo tengo que reconocer.

Durante el proyecto, se observó que las carencias de conocimientos en IA en los docentes se relacionan con la necesidad de: a) una fundamentación epistemológica de la problemática identificada, b) una intervención fundamentada y sistematizada para combatir la problemática, c) estrategias de colaboración y reflexión con pares del contexto educativo, d) técnicas pertinentes para documentar la experiencia de intervención, su impacto y sus limitantes, e) procedimiento de análisis de datos, y f) estrategias de socialización de los resultados.

\section{Experiencia con proyectos de investigación}

En las escuelas normales se observó que las políticas institucionales están condicionando a los profesores a realizar investigación y generar productos de tales proyectos. Como lo indican Humberto y otros profesores, ante tal requerimiento, han desarrollado estrategias para contar con proyectos comunes. Estos proyectos les permiten generar ponencias, memorias y capítulos para mantener un estatus institucional. En los grupos focales y talleres los participantes externaron que en los proyectos indagan problemáticas del contexto educativo, recabando datos numéricos a través de cuestionarios, rúbricas y análisis de calificaciones.

Humberto: [...] el mismo proceso que hemos tenido en los últimos cinco años, bueno tres cuatro años, metiéndonos un poquito más formal [en el desarrollo de investigaciones], por las exigencias del perfil PRODEP y todas las cuestiones, querer entrar en un cuerpo académico [...].

Diólo pos sobre Educación año 11 | número 21 | julio-diciembre 2020 | ISSN 2007-2171 
Con respecto a la realización de proyectos de IA, como lo señala Elena, se promueve entre los estudiantes como una estrategia de titulación o para favorecer la capacidad reflexiva que requerirán en su futura vida profesional. No obstante, los propios profesores de las escuelas normales no la utilizan para fortalecer su perfil docente. Como se discutió en la sección anterior, los docentes reconocen que les es difícil implementar la IA para analizar su propia práctica docente debido a la falta de conocimientos en relación con la puesta en marcha y sistematización de los diferentes ciclos de la IA. Pero otros, como lo señala Gimena, no conciben la investigación como un aspecto relevante de su actividad como docente normalista.

Elena: Hemos intentado, bueno, he intentado hacer mis pininos con los chicos que deciden titularse por tesis, pero en sí, no tengo una consolidación firme en esta área [la realización de proyectos de IA].

Gimena: No es significativo [el desarrollo de proyectos de investigación acción], o algo dedicado a la docencia [...] entonces son dos actividades que sé, que estoy consciente que, si lo hiciera, el nivel académico sería mejor, pero en eso le digo que siento que no lo valoro tanto, por eso no he tenido el avance que se requiere.

\section{Expectativas de la IA}

Los profesores reconocieron que la IA puede ayudarles a mejorar su propia práctica educativa y desarrollar bases epistemológicas y metodológicas para analizar y trasformar su quehacer docente. A su vez, los profesores consideraron que la articulación entre las escuelas normales y las universidades estatales tiene un impacto bidireccional en los proyectos de ambas instituciones, como lo indica Walter. Los profesores consideran que sus pares de las universidades contribuyen a fortalecer el andamiaje epistemológico y metodológico de los proyectos realizados en la Escuela Normal. A su vez, los profesores normalistas apoyan a sus pares universitarios en la identificación de problemas del quehacer docente en las escuelas públicas.

Walter: Es muy interesante poder coadyuvar en lo que se hace dentro de una escuela normal en comparativo en lo que se hace en la UJAT. Por ejemplo, en las cuestiones de la enseñanza aprendizaje creo que sería una investigación muy interesante para lo que se está trabajando en ambas instituciones.

Un aspecto interesante en la percepción de los participantes durante todo el proyecto fue que sus expectativas de la IA se limitaron exclusivamente a la mejora de la práctica educativa. Durante la interacción entre investigadores e investigados, los docentes normalistas no logra- 
ron vislumbrar que la IA puede, además de ayudarles a mejorar su práctica educativa, proveerles insumos para generar productos académicos que fortalezcan sus perfiles profesionales y el estatus de su CA.

\section{Desafíos para la implementación de la IA}

Durante la fase inicial del proyecto, la noción de desafíos en el empleo de la IA no fue evidente para los docentes. Al inicio del proyecto, solo un desafío fue perceptible: la necesidad de desarrollar conocimientos y habilidades metodológicas en IA a nivel individual. No obstante, durante la segunda fase del proyecto, cuando los docentes ya debían estar implementando la IA, comenzaron a hacerse evidentes diversos retos para desarrollar la IA.

Como lo expresan Claudia y Lourdes, las dinámicas de interacción interpersonal desempeñan un papel determinante en la realización de la IA. Por una parte, la colegialidad y las relaciones de amistad al interior del CA favorecen el trabajo de IA. Por otra, como lo expresa Diana, diversos docentes carecen de interés en realizar actividades ajenas a la docencia. Estos puntos de vista antagónicos dificultan la integración de equipos de trabajo con profesores externos al CA.

Claudia: Realmente el cuerpo académico somos un grupo de compañeros y de amigos que tenemos muchos años, que son muchos años trabajando juntos y generalmente nos organizamos y trabajamos juntos [...] hay maestros que si no se refleja en su bolsillo no tiene utilidad [...].

Lourdes: Va hacer difícil mover un poco de tradiciones que hay en la institución porque hay maestros que ya [...] traen una forma de trabajar [...].

Diana: He visto solamente gente que llega a sus horas frente a grupo, esto de la investigación acción requiere un compromiso y no es nada más llegar a las horas frente a grupo, es intentar colegiar con los otros, revisar materiales, hacer una propuesta, probarla en el aula y es a lo mejor optimizar mis tiempos en la institución con los estudiantes, a lo mejor quedarme un poquito más de tiempo, dos o tres horas antes de mi clase y son cosas que yo no veo.

A nivel institucional, los desafíos para la favorecer el desarrollo docente mediante la IA se encuentran estrechamente vinculados con la visión y las políticas que los directores en cada escuela normal realizan de manera discrecional. Al respecto, la falta de visión y estrategia institucional en las escuelas de Salvador y Lourdes contrastan con la visión de las directoras de la escuela normal de Claudia.

Lourdes: Nos deben dedicar dentro del horario de los docentes que son parte de este proyecto, desde su escuela, un espacio para dedicarse a esto, que tengamos institucionalmente el espacio para organizarme y dedicar horas específicas para el proyecto...

\section{Diólo@os}


Salvador: Les preguntaba a otros cuerpos académicos, si ellos tenían un día asignado [para la investigación], y dicen que sí, y el semestre pasado lo teníamos nosotros asignado y ellos no tenían... No creo que lo roten, sino que, le digo que es parte de una lucha... cuando se hacen los horarios ya la misma dirección diga: sabes que, [...] estas son las horas que tú le debes dedicar a la investigación [...].

Claudia: La directora en ese momento gestionó [...] que pudieran asignarnos a nuestros horarios dos horas semanales... En la administración posterior, la directora de la escuela pues estaba muy metida en las cuestiones de investigación, aunque no se pudo rescatar lo de las horas asignadas, ella daba todas las facilidades para que se pudieran hacer todas las reuniones... En ambos casos, las dos estaban completamente seguras de que lo que estaban haciendo era por el bien de la institución y [...] ven que también en colectivo hay beneficios.

\section{Discusión}

Los cambios curriculares y las políticas educativas enfatizan la autonomía del profesor para trasformar su práctica educativa y contribuir a su desarrollo docente (Arias y Restrepo, 2009), y los docentes normalistas participantes en este estudio se mostraron conscientes de esta realidad. A diferencia de los resultados en otros estudios (Foreman-Peck y Murray, 2008; Kayaoglu, 2015), nuestros participantes manifestaron interés en la IA para lograr la autonomía y la trasformación de su práctica educativa, aun cuando su empleo surgió de una estrategia centralizada. No obstante, al igual que en otros estudios (Flessner y Stuckey, 2014; Krell, 2012), solo al involucrarse directamente con ella lograron identificar varios retos profesionales, personales e institucionales.

El primer reto observado para la implementación de la IA lo constituye la necesidad de los participantes para articular la investigación y la docencia, empleando un plan de intervención sustentado ontológica, axiológica, epistemológica y metodológicamente (Latorre, 2003; Elliot, 2005; Burns, 2010). En su estado actual, los pocos trabajos de investigación que realizan los profesores del estudio poseen un carácter descriptivo cuya función primordial es cuantificar las causas de las problemáticas estudiadas. Por su carácter descriptivo, carecen de un plan de acción con estrategias para subsanar la problemática investigada empleando procedimientos cuya efectividad y las limitaciones sean registradas y analizadas sistemáticamente.

Para lograr tales registros y análisis, los participantes requieren conocimientos declarativos y procedimentales de IA, al igual que otros docentes en servicio y en etapas tempranas de formación investigativa (Addler, 2003; Ulvik y Riese, 2015). Tal necesidad proporciona evidencias empíricas a las conjeturas realizadas en indagaciones anteriores (Vilchis, 2015) y diagnósticos institucionales (Malo, 2017, 2018). Aun cuando existen algunos docentes que expresan poco interés en el desarrollo de tales conocimientos, otros participantes están deseosos de subsanar la falta de estos conocimientos debido a las expectativas que tienen de la IA (Foreman-Peck y Murray, 2008).

En las escuelas normales del estudio, las necesidades observadas permiten identificar tres perfiles docentes. El primero tiene cierta claridad en cuanto a dos objetivos de la IA: la iden- 
tificación de una problemática en su práctica educativa y la solución a tal problemática desde el aula. No obstante, es necesario desarrollar conocimientos y habilidades para construir tanto el andamiaje epistemológico de la problemática como el andamiaje metodológico para combatirla a través de acciones trasformadoras. El segundo requiere aclarar confusiones, tanto epistemológicas como procedimentales, acerca de los diversos tipos de investigación educativa. Finalmente, existe un tercer grupo de docentes que carece incluso de conocimientos básicos de investigación educativa.

Para varios participantes, la curiosidad profesional es un aspecto que incentiva su interés en la IA. Para otros, la IA es un requisito de política educativa nacional que están dispuestos a cubrir. Independientemente de estos posicionamientos, los docentes normalistas visualizaron la IA como un proceso que les permite trasformar su práctica educativa cotidiana. No obstante, su percepción y expectativas de la IA permanecen limitadas ya que no consideran que puede proveerlos de insumos necesarios para fortalecer su desarrollo profesional (Foreman-Peck y Murray, 2008; Flessner y Stuckey, 2014; Kayaoglu, 2015), generar productos científicos (Colmenares y Piñero, 2008; Tanner y Davies, 2009; Burns, 2010) y mejorar el estatus de su CA.

Esta limitante se explica desde la percepción que tienen de la IA como una estrategia de intervención docente, desarticulada de un proceso sistematizado de recolección y análisis de datos (Addler, 2003). También puede estar vinculada con la falta de fundamentación en sus reflexiones y la presentación de sus hallazgos (loannidou y Patsalidou, 2015; Luttenberg, Meijer y OolbekkinkMarchand, 2016). Como lo señalan varios investigadores educativos (Becerra y Moya, 2010; Colmenares y Piñero, 2015), cuando las experiencias de innovación en el aula se documentan y analizan apegándose al principio de sistematización, la IA provee a los docentes con datos y resultados valiosos para los actores de sus contextos educativos y la comunidad científica.

Aunado a la carencia de conocimientos, existen dinámicas personales en cada contexto educativo que resultan en detrimento de la IA (Ioannidou y Patsalidou, 2015). En el contexto del estudio, la IA se desarrolla básicamente por tres grupos de docentes que comparten afinidades personales. Independientemente de la estrategia institucional, la negatividad de sus colegas docentes los llevó a agruparse en cuerpos académicos para mejorar entre ellos su quehacer docente y desarrollar conocimientos y habilidades que incidan en su práctica educativa. Desde esta mirada, la afinidad personal entre ellos les permite asumir obligaciones colegiadas, delegar responsabilidades, compartir sus datos, pero, sobre todo, reflexionar de manera colaborativa, solidaria y empática.

\section{Conclusión}

Este estudio presenta evidencias empíricas para entender algunos desafíos que enfrentan los profesores de las escuelas normales al realizar investigación-acción como una estrategia para la innovación de su práctica pedagógica y el fortalecimiento de sus perfiles profesionales. Al res- 
pecto, se documentaron diversos factores relacionados con el propio docente, al igual que en otros contextos educativos. No obstante, como lo señalan Adler (2003) e loannidou y Patsalidou (2015), diversos aspectos exógenos inciden en el nivel de compromiso que demuestran hacia la IA.

Uno de estos aspectos se relaciona con la discrepancia entre la política institucional de promover la investigación y las acciones discrecionales emprendidas para tal efecto en cada contexto educativo (Krell, 2012). En este sentido, la política institucional se interesa en el desarrollo de proyectos de IA que permitan la generación de productos que fortalezcan el desarrollo del perfil profesional de los docentes normalistas y el grado de consolidación de sus CA. Sin embargo, las acciones emprendidas por las autoridades educativas generan escepticismo para la consolidación de la IA, al igual que en otros contextos (Foreman-Peck y Murray, 2008; Kayaoglu, 2015).

Este escepticismo es el resultado del grado de compromiso organizacional de sus instituciones para apoyar las acciones requeridas durante el desarrollo de la IA (Flessner y Stuckey, 2014). Por ejemplo, se necesita claridad en relación con las acciones concretas que los directores de los centros educativos deben impulsar para promover la IA como una estrategia de desarrollo profesional para todos sus docentes (loannidou y Patsalidou, 2015; Krell y Fichtman, 2012). No obstante, como lo demuestran Foreman-Peck y Murray (2008), tales acciones no deben ser discrecionales para evitar conflictos de interés y favorecer el compromiso personal e institucional. Por el contrario, tales acciones deben ser consensuadas abiertamente entre los administradores y los docentes.

En consecuencia, a nivel organizacional, el éxito en la implementación de la IA como una estrategia institucionalizada requiere de acciones organizativas titánicas y concretas (Kayaoglu, 2015). En estas acciones se requiere claridad y coherencia con respecto a las funciones y compromisos de los diferentes niveles institucionales, administradores y actores educativos. En conjunto, deberán enfrentar de manera colaborativa los desafíos personales, interpersonales e institucionales que vayan surgiendo. De lo contrario, la política educativa, las iniciativas institucionales y la voluntad de los docentes permanecen desarticuladas y alejadas del objetivo de la IA de empoderar al docente para lograr la innovación de su práctica educativa y favorecer su desarrollo profesional.

\section{Agradecimientos}

Agradecemos la invaluable participación de los profesores normalistas, así como el apoyo institucional de las autoridades de las tres escuelas normales que participaron en el estudio, de la DGESU y de la DGESPE. Nuestro agradecimiento a los editores y evaluadores de la revista Diálogos sobre educación; sus observaciones nos permitieron enriquecer el escrito. El estudio se 
realizó con financiamiento PRODEP, otorgado durante el periodo 2017-2019 al CAC Investigación e Innovación Educativas, de la Universidad Juárez Autónoma de Tabasco, a través del proyecto con clave de registro UJA-CA-245.

\section{Referencias}

Addler, S. (2003). Dilemmas of Action Research. Action in Teacher Education, 25, 76-82.

Arias, C.; M. Restrepo (2009). La investigación-acción en educación: un camino hacia el desarroIlo profesional y la autonomía. Íkala, Revista de Lenguaje y Cultura, 14(22), 109-122.

Balcázar, P.; N. González; G. Gurrola; A. Moysén (2015). Investigación cualitativa. México: Universidad Autónoma del Estado de México.

Becerra, R.; A. Moya (2010). Investigación-acción participativa, crítica y transformadora. Un proceso permanente de construcción. Integra Educativa, 3(2), 133-156.

Burns, A. (2010). Doing action research in English language teaching. A guide to practitioners. Nueva York: Routledge.

Colmenares, A.; L. Piñero (2008). La investigación acción. Una herramienta metodológica heurística para la comprensión y transformación de realidades y prácticas socio-educativas. Laurus, 14(27), 96-114.

Creswell, J. (2013). Qualitative inquiry and research design. Choosing among five approaches. Thousand Oaks: Sage.

Dick, B. (2010). Action research literature 2008-2010. Themes and trends. Action Research, (9), 122-143.

Dörney, Z. (2002). Questionnaires in second language research. Construction, administration, and processing. Nueva York: Routledge.

Elliott, J. (2005) Becoming Critical: The Failure to Connect. Educational Action Research, 13, 359373.

Flessner, R.; S. Stuckey (2014). Politics and education research: An examination of one school's mandated action research program. Action Research, 12, 36-51.

Foreman-Peck, L.; J. Murray (2008). Action research and policy. Journal of Philosophy of Education, 42, 145-163.

Imbernón, F. (coord.) (2000). La investigación educativa como herramienta de formación del profesorado. Reflexión y experiencias de investigación educativa. Barcelona: Graó.

Ioannidou, M.; F. Patsalidou (2015). Engaging school teachers and school principals in an action research in-service development as a means of pedagogical self-awareness. Educational Action Research, 23, 124-139.

Kayaoglu, N. (2015). Teacher researchers in action research in a heavily centralized education system. Educational Action Research, 23, 140-161.

Diálo@os año 11 | número 21 | julio-diciembre 2020 | ISSN 2007-2171 
Krell, D.; N. Fichtman (2012). Facilitating action research: A study of coaches, their experiences, and their reflections on leading teachers in the process of practitioner inquiry. Professional Development in Education, 38, 827-844.

Latorre, A. (2003). La investigación-acción. Conocer y cambiar la práctica educativa. Barcelona: Graó.

Luttenberg, J.; P. Meijer; H. Oolbekkink-Marchand (2016). Understanding the complexity of teacher education in action research. Educational Action Research, 25, 88-102.

Malo, S. (2017). Estrategias de fortalecimiento y transformación de las escuelas normales. Proyecto RECREA. En Urbano, G. (Directora de PRODEP). Primer Seminario Taller RECREA, Cd. México, 2017. México: PRODEP.

Malo, S. (2018). Estrategias de fortalecimiento y transformación de las escuelas normales. Proyecto RECREA. En Pedroza, R. (Líder de la Red RECREA). Segundo Seminario Taller RECREA, Toluca, 2018. México: Universidad Autónoma del Estado de México.

Órgano de Gobierno Constitucional de los Estados Unidos Mexicanos (2012). Diario Oficial de la Federación. Tomo DCCVII. No.14. México: Gobierno Constitucional de los Estados Unidos Mexicanos.

Secretaría de Educación Pública (1997). Plan de Estudios. Licenciatura en Educación Primaria. México: SEP.

Tanner, H.; S. Davies (2009). How engagement with research changes the professional practice of teacher-educators: A case study from the Welsh Education Research Network. Journal of Education for Teaching: International Research and Pedagogy, 35(4): 373-389.

Thouin, M. (2014). Réaliser une recherche en didactique. Montreal: Éditions Multimondes.

Ulvik, M.; H. Riese (2015). Action research in pre-service teacher education - a never-ending story promoting professional development. Professional Development in Education, 42, 441457.

Vaughan, M.; G. Burnaford. (2015). Action research in graduate teacher education: A review of the literature 2000-2015. Educational Action Research, 24, 280-299.

Vilchis Pérez, K. (2015) Investigación en la formación docente. Una mirada desde el currículo. Revista Iberoamericana para la Investigación y el Desarrollo Educativo, 5(10), 147-159. 